\title{
Via de parto: influência no teor de gorduras do colostro de nutrizes em maternidade do interior do Estado de São Paulo
}

\author{
Mode of delivery: influence on the fat content of the colostrum of nursing mothers in a maternity \\ hospital in the interior of the State of São Paulo \\ Modo de parto: influencia en el contenido graso del calostro de madres lactantes en una \\ maternidad del interior del Estado de São Paulo
}

Recebido: 19/01/2021 | Revisado: 25/01/2021 | Aceito: 30/01/2021 | Publicado: 06/02/2021

Letícia Aguiar de Campos

ORCID: https://orcid.org/0000-0003-4011-3520 Universidade do Oeste Paulista, Brasil

E-mail: le_aguiar_@hotmail.com Jossimara Polettini

ORCID: https://orcid.org/0000-0002-6719-8715 Universidade Federal da Fronteira Sul, Brasil E-mail: jossimara.polettini@uffs.edu.br

Lucas Lima de Moraes

ORCID: https://orcid.org/0000-0003-1613-5068 Universidade Estadual de Londrina, Brasil

E-mail: lucaslima16@outlook.com

Bruna Decco Marques da Silva

ORCID: https://orcid.org/0000-0002-9595-9446

Universidade Estadual de Londrina, Brasil

E-mail: bruna.decco@hotmail.com

Erika Kushikawa Saek

ORCID: https://orcid.org/0000-0002-8604-0374 Instituto Adolfo Lutz-IAL/Presidente Prudente, Brasil

E-mail: erikaksaeki@gmail.com

Denise Vasconcelos de Jesus Ferrari

ORCID: https://orcid.org/ 0000-0003-1365-4823

Universidade do Oeste Paulista, Brasil E-mail: denisevj@hotmail.com

Glilciane Morceli

ORCID: https://orcid.org/0000-0001-8216-9931

Universidade do Estado de Minas Gerais, Brasil E-mail: glilciane.morceli@uemg.br

\begin{abstract}
Resumo
Objetivo: comparar o teor de gorduras do colostro em nutrizes com a via de parto e descrever as características gestacionais. Trata-se de um estudo transversal, no qual foram incluídas nutrizes que tiveram parto vaginal ou cesárea, atendidas em uma maternidade de Presidente Prudente de dezembro de 2017 a abril de 2018. A coleta foi realizada por ordenha manual entre 48 e 72 horas após o parto para a dosagem de gorduras pela técnica do crematócrito. Dados pessoais, comportamentais, obstétricos/ gestacionais foram coletados seguindo protocolo estabelecido para o estudo e análise dos prontuários médicos. Todas as nutrizes foram informadas quanto à finalidade da pesquisa e assinaram o termo de consentimento livre e esclarecido. As análises estatísticas foram realizadas utilizando-se teste t, ou teste $\mathrm{Z}$ ou teste de Mann-Whitney. Para todos os testes o nível de significância adotado foi de 5\%. Foram descritos características sócios demográficos como idade materna estatisticamente inferior nas nutrizes que vivenciaram o parto vaginal $(\mathrm{p}<0,005)$, gestacionais e obstétricas e resultados perinatais dos recém-nascidos $(\mathrm{RN})$ de parto vaginal e cesárea. A via de parto vaginal ou cesárea não influenciaram na composição do teor de gorduras do colostro.
\end{abstract}

Palavras-chave: Parto normal; Cesárea; Colostro; Amamentação.

\begin{abstract}
Objective: To compare the fat content of colostrum in nursing mothers with the method of delivery and to describe the gestational characteristics. This is a cross-sectional study including mothers who underwent vaginal delivery or cesarean delivery, attended at a maternity ward in Presidente Prudente from December 2017 to April 2018. The collection was performed through manual milk expressing between 48 and 72 hours postpartum for the dosage of fats, using the crematocrit technique. Personal, behavioral, and obstetrical/gestational data were collected following a
\end{abstract}


protocol established for the study and analysis of medical records. All the nursing mothers were informed about the purpose of the research and signed the informed consent form. Statistical analyzes were performed using the t-test, Ztest, or Mann-Whitney test. For all tests, the level of significance was 5\%. Demographic characteristics were described, such as the maternal age was statistically lower in mothers who underwent vaginal delivery $(\mathrm{p}<0.005)$, gestational and obstetric, and perinatal outcomes of newborns (NB) from vaginal and cesarean delivery. Vaginal or cesarean delivery did not influence the composition of the colostrum fat content.

Keywords: Normal delivery; Cesarean section; Colostrum; Breastfeeding.

\section{Resumen}

Objetivo: Comparar el contenido graso del calostro en madres lactantes con el método de parto y describir las características gestacionales. Se trata de un estudio transversal que incluyó a madres sometidas a parto vaginal o cesárea, atendidas en una maternidad de Presidente Prudente desde diciembre de 2017 a abril de 2018. La recolección se realizó mediante extracción manual de leche entre las 48 y 72 horas posparto para la dosificación de grasas, mediante la técnica del crematocrito. Los datos personales, conductuales y obstétricos / gestacionales se recolectaron siguiendo un protocolo establecido para el estudio y análisis de las historias clínicas. Todas las madres lactantes fueron informadas sobre el propósito de la investigación y firmaron el formulario de consentimiento informado. Los análisis estadísticos se realizaron utilizando la prueba t, la prueba $\mathrm{Z}$ o la prueba de Mann-Whitney. Para todas las pruebas, el nivel de significancia fue del 5\%. Se describieron características demográficas, como la edad materna fue estadísticamente menor en las madres que se sometieron a parto vaginal $(\mathrm{p}<0,005)$, los resultados gestacionales y obstétricos y perinatales de los recién nacidos $(\mathrm{RN})$ por parto vaginal y cesárea. El parto vaginal o por cesárea no influyó en la composición del contenido de grasa del calostro.

Palabras clave: Parto normal; Cesárea; Calostro; Lactancia.

\section{Introdução}

A partir do terceiro trimestre gestacional há uma elevação nos níveis de citocinas pró-inflamatórias principalmente interleucinas (IL) IL-1 e IL-8 no microambiente materno-fetal, que estimulam o aumento nos níveis de prostaglandina, resultando em contrações uterinas, caracterizando o início do trabalho de parto (Challis et al., 2009; Shynlova et al., 2012). Para que se estabeleça o trabalho de parto, é necessária a ocorrência de cinco eventos fisiológicos: ruptura das membranas fetais, amadurecimento e dilatação uterina, contratilidade do miométrio, dequitação da placenta e involução uterina (Norman et al., 2007; Reti et al., 2007). No entanto, em algumas gestantes, as alterações do sistema imune e endócrino não acontecem espontaneamente quando a gestação chega ao termo, ou seja, com mais de 37 semanas de gestação, quando se considera que exista a maturidade fetal para o nascimento (Kamel, 2010). Assim, o parto induzido por medicamentos ou a realização de intervenção cirúrgica para retirada do feto podem ser indicados.

A cesárea é indicada em casos específicos quando há comorbidades maternas, como incompetência istmo-cervical, diabetes, obesidade e hipertensão arterial (Barroso Zimmermmann et al., 2008). Ainda, a cesárea é indicada em detrimento ao uso de instrumentos que forcem o parto vaginal, como fórceps e sistema a vácuo, em mulheres em trabalho de parto, ou seja, com contrações uterinas vigentes, mas sem dilatação cervical suficiente. O fórceps e o sistema a vácuo elevam as taxas de hemorragias e anemia severa materna em relação à cesárea (Polkowski et al., 2017).

Os avanços em relação às técnicas cirúrgicas nas últimas décadas propiciaram uma diminuição considerável nas intercorrências decorrentes da cesárea para o binômio mãe/recém-nascido, e, culturalmente, esse procedimento tem sido concebido como um salva-vidas para ambos. Além disso, a comodidade de ter um parto agendado e momentaneamente sem dor aumentaram os índices de cesáreas nas últimas décadas (Mandarino et al., 2009). Em trabalho recente, 60\% das gestantes questionadas sobre os motivos que as levariam a trocar o parto natural pelo cirúrgico indicaram a praticidade como principal fator e quase 56\% indicaram o medo de sofrimento e dor (Leguizamon Junior et al., 2013). Nesse contexto, Gregory et al, 2012 ressaltam que as dificuldades em avaliar a compensação do risco-benefício tem sido um desafio, pois durante a decisão do tipo de parto pela equipe de atendimento juntamente com a mãe nem sempre os fatores de riscos ficam explícitos, e a escolha muitas vezes é baseada na conveniência e na cultura do local e não considerando que a evolução da gestação deve seguir o curso para parto vaginal, a menos que haja uma razão médica para uma cesariana, conforme orientação do Ministério da Saúde 
do Brasil (Brasil, 2015).

Quando a cesárea é realizada em casos em que o parto vaginal poderia progredir sem maiores riscos para a gestante, a cesárea pode provocar consequências adversas tanto para mãe quanto para o recém-nascido o que tem sido alvo de muitos trabalhos na literatura. Aproximadamente 2,7\% das gestantes que experimentam a cesárea eletiva (planejada, de caráter não emergencial) apresentam morbidades severas, taxa expressivamente superior a $0,9 \%$ observada em gestantes com experiência de parto vaginal (Liu et al.,2007). Wang et al., verificaram aumento do risco de desenvolvimento de leucemia linfoblástica aguda em neonatos nascidos de cesárea eletiva em comparação a gestantes com parto vaginal. Além disso, os riscos da cesariana a curto prazo incluem aumento do risco de infecção, complicações anestésicas e cirúrgicas, principalmente hemorragias (revisado por Gregory et al, 2012). Em especial atenção, está a menor probabilidade de amamentar ou cessação do aleitamento até nas primeiras horas de vida do neonato nos casos de partos cirurgicamente induzidos, o que pode ter consequências tardias na vida do recém-nascido (Barroso Zimmermmann et al., 2008). Além da diminuição da quantidade de leite materno produzido e do tempo total de aleitamento, a cesárea parece influenciar na composição do leite, em particular no colostro (Şimşek et al., 2014, Morceli et al., 2010). A princípio, pode-se destacar a diferença na composição da microbiota no leite materno advindo de gestantes com diferentes tipos de parto (Khodayar-Pardo et al., 2014). Em relação aos nutrientes, Dizdar et al, 2014 reportaram que os níveis proteicos no colostro de mães que tiveram parto vaginal foram estatisticamente superiores aos níveis do colostro de mães que tiveram cesárea. Simsek et al, 2015 descreveram que os níveis de antioxidantes totais nessa primeira secreção láctea são significativamente maiores quando provenientes de gestações com parto vaginal em comparação com cesárea. O estresse oxidativo tem sido associado a casos de parto pré-termo e resultados neonatais adversos (Leal et al., 2011; Gelaleti et al., 2015).

A concentração de gorduras aumenta durante a amamentação, assim o leite final da mamada (leite posterior) é mais rico em energia (Brasil, 2009). Lubetzky et al., 2015 relataram sobre a influência da idade materna avançada no aumento da concentração de gorduras o que difere do estudo realizado por Moraes et al., 2019 que evidenciaram que não há correlação entre idade materna e concentração de gordura. No entanto, o significado biológico desses achados permanece desconhecido.

Há na literatura estudos que relacionam a gordura do colostro de nutrizes associado à patologias durante o período gestacional, tais como gestações complicadas por diabetes e obesidade (Morceli et al., 2010; Fujimori et al., 2015).

Ferrari et al., $2020 \mathrm{em}$ seu estudo correlacionou a idade materna com o perfil de citocinas do leite humano de nutrizes que amamentam e não evidenciou diferença entre os grupos.

Granot et al., 2016 e Kovács et al., 2005 não encontraram diferenças significativas no teor de gordura entre RN prétermo quando comparados com $\mathrm{RN}$ a termo.

Assim, o objetivo do presente estudo foi comparar o teor de gorduras do colostro de nutrizes com a via de parto vaginal e/ou cesárea no termo (idade gestacional $\geq 37$ semanas) e descrever as características gestacionais e os aspectos sócios demográficos maternos.

\section{Metodologia}

Estudo transversal, de abordagem quantitativa optou-se por esta abordagem por tratar-se de um método que visa a comprovação de hipóteses por meio de quantificações (Soares Pereira et al., 2018). Aprovado pelo Comitê de Ética em Pesquisa da Universidade do Oeste Paulista, UNOESTE, via Plataforma Brasil, (55688216.8.0000.5515), todas as nutrizes assinaram o Termo de Consentimento Livre e Esclarecido (TCLE) e foram atendidas na maternidade do Hospital Regional de Presidente Prudente no período de fevereiro a dezembro de 2017.

Os critérios de inclusão foram a idade gestacional no parto entre 37 a $41^{6 / 7}$ semanas, reações sorológicas negativas para hepatite, HIV e sífilis e ausência de adversidades gestacionais. Foram excluídas as gestantes diagnosticadas com 
malformações fetais e gravidez gemelar.

A estimativa do tamanho amostral considerou o número de partos realizados no serviço no período de um mês, em torno de 120 partos/mês considerando a diferença das médias de parto vaginal e cesárea, pela menor distância entre os resultados e o maior tamanho da amostra. Considerando a estimativa amostral foram incluídas 214 nutrizes, distribuídas nos grupos parto vaginal $(n=131)$ e no grupo parto cesárea $(n=83)$.

Os dados sociodemográficos e obstétricos foram coletados a partir de entrevista e questionário próprio do estudo e análise dos prontuários médicos.

O colostro foi coletado manualmente, com técnica adequada, sempre no período da manhã e no intervalo entre duas mamadas, no período correspondente entre 48 a 72 horas após o parto. A quantidade máxima de colostro coletada foi de $10 \mathrm{ml}$. Essas técnicas seguiram as orientações protocolares das boas práticas clínicas. Após a coleta as amostras foram armazenadas a $-20^{\circ} \mathrm{C}$ e posteriormente submetidas aos métodos de dosagens.

A determinação do teor de gorduras foi obtida pelo método do crematócrito descrito por Lucas et al., 1978. As amostras foram aquecidas em banho-maria a $40^{\circ} \mathrm{C}$ por 15 min e submetidas à mistura em vórtex. Colocadas em tubos capilares $(2 \mu \mathrm{l})$, preenchidos a aproximadamente três quartos de amostra, selados com lacre e centrifugados por 15 min. Após centrifugação ocorreu à separação entre o creme e o soro do leite. Foram aferidos o comprimento da coluna de creme e coluna total do produto.

$\mathrm{O}$ teor de gordura e calorias foram calculados pelas seguintes fórmulas: 1 . Teor de Gordura $=\%$ creme $(\mathrm{mm})-0.59$ / 1.46 , onde a $\%$ creme $=$ coluna de creme $(\mathrm{mm}) \times 100 /$ coluna total $(\mathrm{mm}) ; 2 . \mathrm{Kcal} / \mathrm{L}=(66.8 \times \%$ creme $)+290$.

Os dados sociodemográficos e obstétricos foram armazenados em planilhas do software Excel 2010, e compilados para a análise das variáveis estudadas utilizando se teste de comparações de Mann-Whitney, X2 ou exato de Fisher. Todos os testes foram realizados em software Graph Pad Prism, versão 6,0 e o nível de significância adotado foi de 5\%

\section{Resultados}

Os resultados são referentes às coletas dos partos vaginais ou cesáreas de 214 nutrizes. Dados sócios demográficos, gestacionais e obstétricos e perinatais dos RN foram descritos nas tabelas 1 e 2. As dosagens de gorduras e calorias foram apresentadas em box plot nas Figuras 1, 2 e 3.

Na Tabela 1 estão descritas as características sócias demográficas, gestacionais e obstétricas das nutrizes com parto vaginal e/ou cesárea. A mediana da idade materna foi estatisticamente inferior nas nutrizes que vivenciaram o parto vaginal $(\mathrm{p}<0,005)$. As demais variáveis retratadas como etnia, estado civil, profissão, hábito tabagista não foram significativas ( $p>0,05)$. O índice de massa corpórea, no início da gestação foi estatisticamente superior no grupo de cesárea $(p=0,01)$ e o ganho de peso foi maior em nutrizes com parto vaginal $(\mathrm{p}=0,01)$. Variáveis como doença gestacional, paridade, número de consultas no pré-natal, idade gestacional e prematuridade expressaram-se estatisticamente insignificantes ( $p>0,05)$.

A Tabela 2 apresenta resultados perinatais dos recém-nascidos (RN) de parto vaginal e cesárea. O New Ballard em semanas/dias apresentou mediana maior nos $\mathrm{RN}$ de parto vaginal se comparado aos $\mathrm{RN}$ de cesárea ( $\mathrm{p}=0,004)$. Outro resultado estatisticamente relevante foi à classificação do peso, os RN classificados como adequados para idade gestacional (AIG) foram maiores em nutrizes com parto vaginal e os RN grande para idade gestacional (GIG) foram evidenciados no grupo de nutrizes com cesárea $(\mathrm{p}=0,006)$. O perímetro cefálico apresentou-se superior nos $\mathrm{RN}$ de cesárea, se comparado aos $\mathrm{RN}$ de parto vaginal $(\mathrm{p}=0,0002)$.

As Figuras 1, 2 e 3 evidenciam a quantificação da porcentagem de gorduras, creme e calorias do colostro e os resultados não foram significativos ( $p>0,05)$. 
Tabela 1. Características sociodemográficas, gestacionais e obstétricas de gestantes com parto vaginal ou cesárea incluídas no estudo.

\begin{tabular}{|c|c|c|c|}
\hline Característica & $\begin{array}{l}\text { Parto Vaginal } \\
\quad(n=131)\end{array}$ & $\begin{array}{l}\text { Cesárea } \\
(\mathrm{n}=\mathbf{8 3})\end{array}$ & 1 \\
\hline \multicolumn{4}{|l|}{ Característica materna } \\
\hline Idade $(\operatorname{anos})^{\mathrm{a}}$ & $24(15-45)$ & $28(12-42)$ & $<0,005 *$ \\
\hline \multicolumn{4}{|l|}{ Etnia $^{b}$} \\
\hline Branca & $65(49,6)$ & $38(45,8)$ & \multirow[t]{2}{*}{0,58} \\
\hline Não Branca & $66(50,4)$ & $45(54,2)$ & \\
\hline \multicolumn{4}{|l|}{ Estado civil ${ }^{b}$} \\
\hline União estável & $100(76,3)$ & $71(85,5)$ & \multirow[t]{2}{*}{0,10} \\
\hline Solteira & $31(23,7)$ & $12(14,5)$ & \\
\hline \multicolumn{4}{|l|}{ Profissão $^{b}$} \\
\hline Remunerada & $41(31,3)$ & $24(28,9)$ & \multirow{2}{*}{0.71} \\
\hline Não remunerada (do lar, estudante) & $90(68,7)$ & $59(71,1)$ & \\
\hline \multicolumn{4}{|l|}{ Hábito tabagista ${ }^{b}$} \\
\hline Sim & $12(9,1)$ & $11(13,2)$ & 0.37 \\
\hline \multicolumn{4}{|l|}{ Características Gestacionais } \\
\hline \multicolumn{4}{|l|}{ Índice massa corpórea (IMC) ${ }^{\mathrm{a}}$} \\
\hline Início gestação & $23,1(14,3-42,9)$ & $25,1(14,8-45,1)$ & $0.01 *$ \\
\hline Final gestação & $27,5(19,2-42,9)$ & $29,7(20,0-51,4)$ & 0.07 \\
\hline Ganho de Peso $(\mathrm{kg})^{\text {a }}$ & $12(-4,0-21,9)$ & $9,3(-4,3-32,0)$ & $0.01 *$ \\
\hline \multicolumn{4}{|l|}{ Doença Gestacional ${ }^{\# ~ b}$} \\
\hline Sim & $55(42,0)$ & $38(45,8)$ & 0,58 \\
\hline \multicolumn{4}{|l|}{ Paridade $^{b}$} \\
\hline Primigesta & $61(46,6)$ & $26(31,3)$ & 0.06 \\
\hline Secundigesta & $32(24,4)$ & $22(26,5)$ & \\
\hline Multípara & $38(29,0)$ & $35(42,2)$ & \\
\hline \multicolumn{4}{|l|}{ Número consultas Pré-Natal ${ }^{\text {b }}$} \\
\hline$\leq 7$ consultas & $35(26,7)$ & $25(30,1)$ & 0,64 \\
\hline$>7$ consultas & $96(73,3)$ & $58(69,9)$ & \\
\hline \multicolumn{4}{|l|}{ Características Obstétricas } \\
\hline $\begin{array}{l}\text { Idade gestacional no parto (semanas, } \\
\text { dias) }^{\mathrm{c}}\end{array}$ & $39 \mathrm{~s} 02 \mathrm{~d} \pm 1 \mathrm{~s} 1 \mathrm{~d}$ & $39 \mathrm{~s} \pm 1 \mathrm{~s} 2 \mathrm{~d}$ & 0,07 \\
\hline \multicolumn{4}{|l|}{ Prematuridade $(<37 \text { semanas })^{b}$} \\
\hline Sim & $1(0,7)$ & $3(3,6)$ & 0,3 \\
\hline
\end{tabular}

a Teste Mann-Whitney, mediana (mínimo - máximo)

b Teste $\mathrm{X}^{2}$ ou Teste exato de Fisher, n (\%) 
c Teste t-student, media \pm DP

* Estatisticamente significativo $(\mathrm{p}<0,05)$

Fonte: Autores

Tabela 2. Resultado perinatais dos recém-nascidos $(\mathrm{RN})$ de parto vaginal e cesárea.

\begin{tabular}{|c|c|c|c|}
\hline Variáveis & $\begin{array}{l}\text { RN de Parto Vaginal } \\
\qquad(\mathrm{n}=131)\end{array}$ & $\begin{array}{c}\text { RN Cesárea } \\
(\mathbf{n = 8 3})\end{array}$ & $p$ \\
\hline New Ballard (semanas, dias) & 39s $(36 s 6 d-41 s)$ & $38 s(35 s-41 s)$ & $0,004 *$ \\
\hline Peso ao nascer $(\mathbf{K g})^{\mathbf{b}}$ & $3,218 \pm 0,378$ & $3,295 \pm 0,528$ & 0,21 \\
\hline \multicolumn{4}{|l|}{ Classificação do Peso ${ }^{c}$} \\
\hline PIG & $6(4,6)$ & $6(7,3)$ & \multirow{3}{*}{$0,006 *$} \\
\hline AIG & $123(93,9)$ & $68(81,9)$ & \\
\hline GIG & $2(1,5)$ & $9(10,8)$ & \\
\hline \multicolumn{4}{|l|}{${ }_{\text {Ápgar de }} 1^{\circ}$ minuto $^{c}$} \\
\hline$\geq 7$ & $124(94,5)$ & $80(96,4)$ & \multirow{2}{*}{0,74} \\
\hline$<7$ & $7(5,5)$ & $3(3,6)$ & \\
\hline \multicolumn{4}{|l|}{${\text { Ápgar de } 5^{\circ} \text { minuto }}^{c}$} \\
\hline$\geq 7$ & $129(98,5)$ & $82(98,8)$ & \\
\hline$<7$ & $2(1,5)$ & $1(1,2)$ & \\
\hline Comprimento $(\mathrm{cm})^{\mathrm{a}}$ & $48,0(35,0-55,0)$ & $48,5(42,5-53,0)$ & 0,23 \\
\hline Perímetro Cefálico $(\mathrm{cm})^{\mathrm{a}}$ & $33,5(31,0-37,0)$ & $34,0(31,5-39,0)$ & $0,0002 *$ \\
\hline Perímetro Torácico $(\mathrm{cm})^{\mathrm{a}}$ & $33,0(29,0-44,0)$ & $33,0(27,0-39,0)$ & 0,21 \\
\hline Perímetro Abdominal $(\mathrm{cm})^{\mathrm{a}}$ & $31,0(26,5-37,0)$ & $31,0(26,0-39,0)$ & 0,29 \\
\hline Índice Ponderal $^{\mathrm{a}}$ & $0,029(0,017-0,283)$ & $0,029(0,021-0,036)$ & 0,75 \\
\hline Dias de Internação ${ }^{a}$ & $2(2-14)$ & $3(2-7)$ & 0,41 \\
\hline
\end{tabular}

a Teste Mann-Whitney, mediana (mínimo - máximo)

b Teste t-student, media \pm DP

c Teste $\mathrm{X}^{2}$ ou Teste exato de Fisher, n (\%), PIG (Pequeno para Idade Gestacional), AIG (Adequado para Idade Gestacional), GIG (Grande para Idade Gestacional).

* Estatisticamente significativo $(\mathrm{p}<0,05)$

Fonte: Autores 
Figura 1. Box plot referente à quantificação da porcentagem de gorduras no colostro de nutrizes após parto vaginal e cesárea. No box-plot, os quadrantes representam de 25 a $75 \%$ dos valores, a mediana está representada pelo traço horizontal e as barras de erro correspondem aos valores mínimos e máximos.

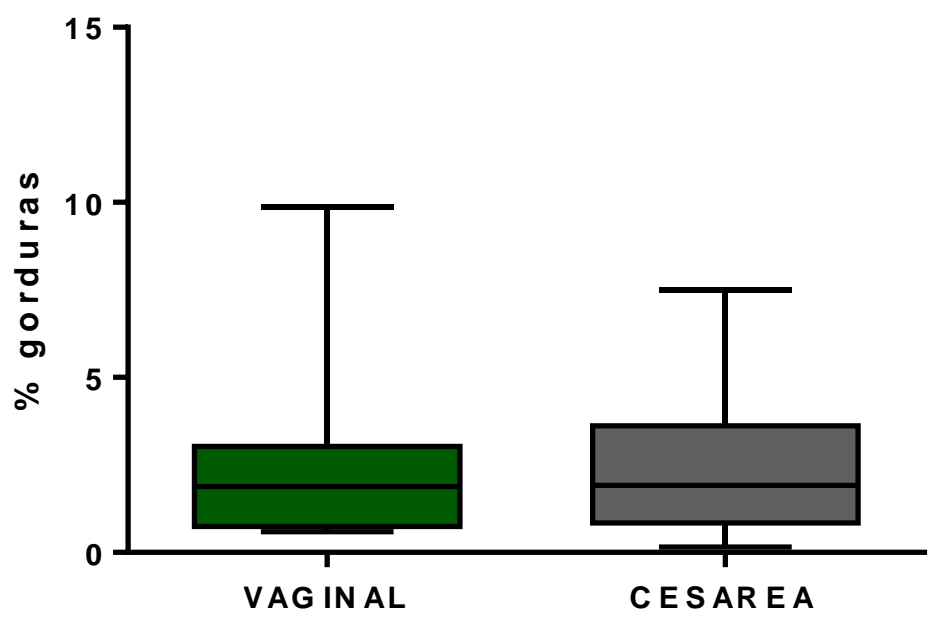

*teste de Mann-Whitney, $\mathrm{p}=0,53$

Fonte: Autores

Figura 2. Box plot referente à quantificação da porcentagem de creme no colostro de nutrizes após parto vaginal e cesárea. No box-plot, os quadrantes representam de 25 a $75 \%$ dos valores, a mediana está representada pelo traço horizontal e as barras de erro correspondem aos valores mínimos e máximos.

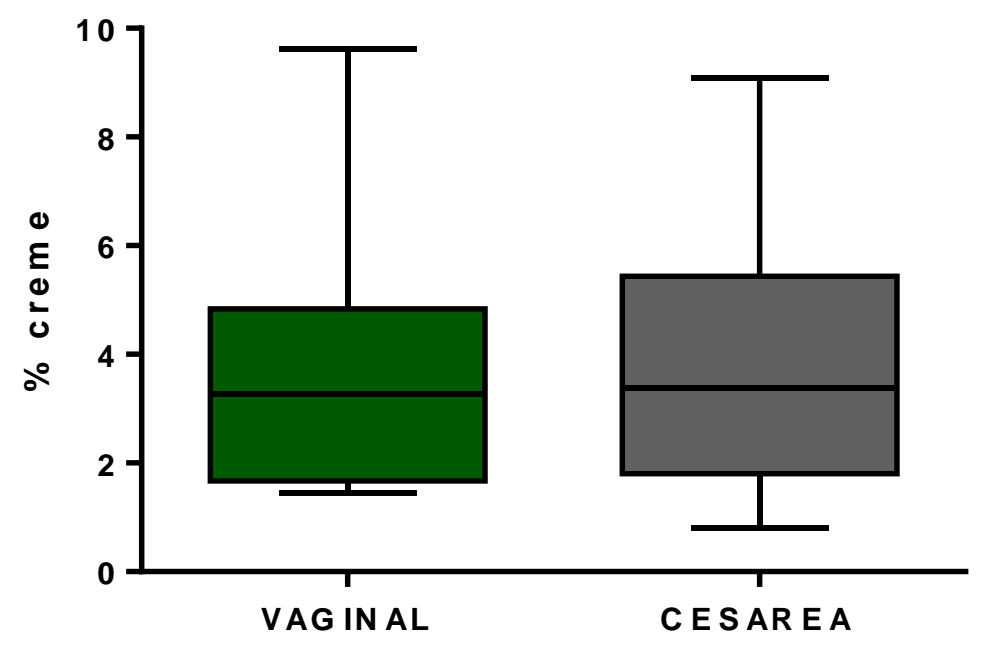

*teste de Mann-Whitney, $\mathrm{p}=0,53$

Fonte: Autores 
Figura 3. Box plot referente à quantificação de calorias no colostro de nutrizes após parto vaginal e cesárea. No box-plot, os quadrantes representam de 25 a $75 \%$ dos valores, a mediana está representada pelo traço horizontal e as barras de erro correspondem aos valores mínimos e máximos.

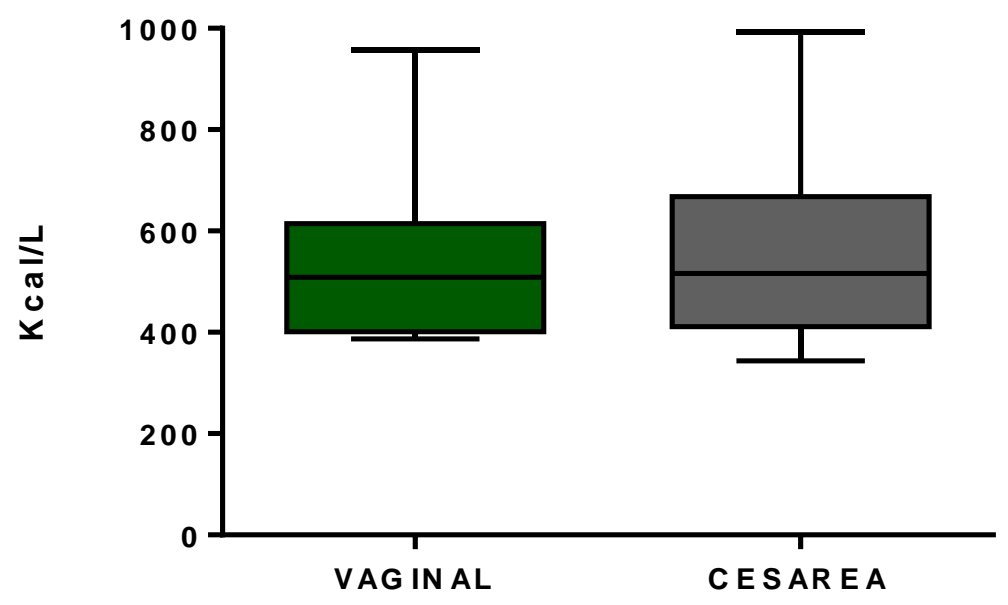

*teste de Mann-Whitney, $\mathrm{p}=0,45$

Fonte: Autores

\section{Discussão}

O presente estudo comparou a composição de gorduras do colostro em nutrizes de parto vaginal e/ou cesárea e descreveu as características sócias demográficas, obstétricas.

Weidle et al., 2014, demonstraram que preferências por determinado tipo de parto estão relacionadas a antecedentes de outros puerpérios ressaltando a importância da escolha do tipo de parto na primeira gestação. Nossos dados mostram que 30 puérperas apresentaram parto vaginal em gestação anterior e 27 com parto cirúrgico prévio. A cesariana pode estar associada a uma barreira para o início do aleitamento materno nas primeiras horas de vida do neonato, relacionada aos cuidados pósoperatórios que ocasionam o retardo ou a interrupção do contato mãe/recém-nascido no período pós-parto (Esteves et al., 2014).

Knupp et al., 2008, evidenciaram que em mais da metade das parturientes consideradas brancas houve predomínio de cesárea. Em contra partida, puérperas negras evoluíram em maior percentil para um parto vaginal. No nosso estudo encontramos dados discrepantes que revelaram $61,21 \%$ de mulheres brancas com parto vaginal. Este achado pode ser devido a amostra ter sido realizada em maternidade pública que preconizam o parto vaginal.

Atualmente muitas mudanças ocorreram na estrutura familiar resultando em um aumento das familias uniparentais, principalmente em mulheres solteiras, ocasionando maior responsabilidade materna. Nosso estudo revelou o predominio de união estável em ambos os grupos. Achados similares aos relatos de Leguizamon et al, 2013 , onde 10\% de puérperas eram solteiras. Esses dados podem estar relacionados pela preferência dos casais em formarem uma união estável e dividirem as responsabilidades.

A profissão predominante nos grupos de estudo foi não remunerada e pode indicar que condições financeiras desfavoráveis associam-se a qualidade da alimentação materna, o que ocasionaria custos elevados para manter uma alimentação equilibrada (Tonini et al., 2013). Segundo Belarmino, 2009, a gestação ocasiona mudanças fisiológicas no organismo da mãe, necessitando de um aumento de nutrientes essenciais, principalmente proteínas, lipídios e carboidratos, para nutrição da própria gestante e garantir crescimento e desenvolvimento do feto. No presente estudo, nutrizes com atividade não remunerada, tiveram resultados não significativos na concentração de creme e gordura no colostro. Contudo a diminuição da 
concentração de gorduras no colostro pode estar associada às condições desfavoráveis, concomitante a má alimentação no período gravídico.

Freire, Sauders, 2009 em seu estudo demonstraram que hábitos tabagistas no período gravídico-puerperal, influenciam na composição do colostro, pois a nicotina pode causar alterações de citocinas presentes no leite, assim como à diminuição da quantidade e volume de leite produzido pela puérpera, o que pode resultar em uma maior susceptibilidade das crianças desenvolverem infecções além de influenciar no crescimento fetal.

Diversos fatores podem influenciar na composição do colostro materno, como dados sócios demográficos, idade, etnia, características gestacionais, assim como índice de massa corporal, paridade, período gestacional, dentre outros. Sinanoglou et al., 2017 demonstraram que dados sócios demográficos podem influenciar na composição do colostro, principalmente relacionados a idade e nacionalidade, e não necessariamente somente sobre a via de parto ou IMC gestacional.

Dourado et al., 2014, avaliaram e quantificaram o teor de proteínas e gorduras no colostro de nutrizes com diferentes IMC, e afirmam que altas concentrações de gorduras no colostro é de extrema importância em função energética para o neonato, e demonstraram que os grupos com IMC alto e IMC normal obtiveram maior concentração de gorduras no colostro se comparado ao grupo com IMC baixo. No nosso estudo pode-se observar que o IMC gestacional no inicio da gestação foi superior na cesárea $(\mathrm{p}=0,01)$. Tal fato não resultou de forma significativa na porcentagem do teor de gorduras do colostro dessas nutrizes, conforme mostra a figura $2(\mathrm{p}=0,53)$. Segundo o autor isso indica que gestantes acima do peso no período gestacional não têm vantagens no teor energético do colostro, porém gestantes abaixo do peso no período gravídico tem maior tendência a produzir colostro com teor energético menor.

Sassa et al., 2014, avaliaram o aleitamento materno (AM) em RN pré-termo, e verificaram que o AM pode ser associado a idade gestacional e ao peso do nascimento, sendo portanto proporcionais, ou seja, quanto menor a idade gestacional e o peso, maior deve ser a ocorrência de amamentação. O AM em neonatos pré-termo, diminui a permanecia no hospital. No presente estudo pode-se observar o New Ballard e classificação do peso AIG superior no grupo de parto vaginal, apresentando um nível de significância ( $\mathrm{p}=0,004)$.

Jaldin et al., 2011, avaliaram o perímetro cefálico em crianças com aleitamento materno exclusivo (AME) nos primeiros seis meses, e pode concluir que o AME, contribuiu no crescimento do perímetro cefálico adequado, sendo portanto fundamental um apoio à nutriz para garantir o aleitamento. Pode-se observar no presente estudo que o perímetro cefálico foi superior no grupo de cesárea $(\mathrm{p}=0,0002)$. Logo, segundo o autor é necessário o AME nos primeiros meses para que o crescimento seja satisfatório para a idade.

A gordura no colostro pode ser influênciada por diversos fatores, incluindo variáveis gestacionais, obstétricas e sócios demograficas. Dizdar et al., 2013 compararam a via de parto, e concluiram que o teor de gorduras presente no colostro é semelhante em ambos. Nota-se que a via de parto por si só não resulta em uma alteração no teor de gorduras, mas sim o conjunto de variáveis durante a gestação.

Estudos semelhantes estão bem eluciados na literatura refletindo variáveis gestacionais/obstétricas, mas a relação entre o teor de gorduras no colostro e a via de parto ainda se mostra escassa.

\section{Conclusão}

A via de parto vaginal ou cesárea não influenciaram na composição do teor de gorduras do colostro. Ressalta-se a importância da realização de mais estudos para elucidar a temática e ainda fortalecer e incentivar o aleitamento materno.

Sugerimos que trabalhos futuros possam aprofundar as possíveis diferenças na composição bioquímica e imunológica do colostro de nutizes com parto vaginal ou cesárea. 


\section{Agradecimentos}

Os autores agradecem a Universidade do Oeste Paulista, e juntamente ao Hospital Regional de Presidente - SP que forneceram estruturas para que este projeto fosse executado. Assim como o Instituto Adolf Lutz por cederem o laboratório de microbiologia para que as análises fossem realizadas.

\section{Referências}

Barroso Zimmermmann, J. Miranda Gomes, C., Soares Pinho Tavares, F., Guimarães Peixoto, I., Campos Vieira de Melo, P., \& Fazzito de Rezende, D. (2008). Complicações puerperais associadas à via de parto. Revista Médica de Minas Gerais,19(2). http://rmmg.org/artigo/detalhes/459

Brasil. Ministério da Saúde. Diretrizes de atenção à gestante: a operação cesariana. Ministério da Saude. 2015; 1-101 .

Brasil. Ministério da Saúde. Saúde da criança: Nutrição infantil, aleitamento materno e alimentação complementar. Cadernos de Atenção Básica, Brasília, DF: Editora MS. 2009.

Belarmino, G. O. Moura, E. R. F., Oliveira, N. C. de, \& Freitas, G. L. de. (2009). Risco nutricional entre gestantes adolescentes. Acta Paulista de Enfermagem, 22(2), 169-175. https://doi.org/10.1590/s0103-21002009000200009

Challis, J. R., Lockwood, C. J., Myatt, L., Norman, J. E., Strauss, J. F., III, \& Petraglia, F. (2009). Inflammation and Pregnancy. Reproductive Sciences, 16(2), 206-215. https://doi.org/10.1177/1933719108329095

Dizdar, E. A., Sari, F. N., Degirmencioglu, H., Canpolat, F. E., Oguz, S. S., Uras, N., \& Dilmen, U. (2013). Effect of mode of delivery on macronutrient content of breast milk. The Journal of Maternal-Fetal \& Neonatal Medicine, 27(11), 1099-1102. https://doi.org/10.3109/14767058.2013.850486

Dourado AA, Sales MF, Alves RR, et al. (2014) Quantificação de proteínas e gorduras do colostro de mulheres com diferentes índices de massa corporal. Rev. Bras. Farm. 95 (1): $499-511,2014$

Esteves, T. M. B., Daumas, R. P., Oliveira, M. I. C. de, Andrade, C. A. de F. de, \& Leite, I. C. (2014). Factors associated to breastfeeding in the first hour of life: systematic review. Revista de Saúde Pública,48(4), 697-708. https://doi.org/10.1590/s0034-8910.2014048005278

Ferrari, D. V. de J., Polettini, J., de Moraes, L. L., de Campos, L. A., da Silva, M. G., Saeki, E. K., \& Morceli, G. (2020). Profile of pro-inflammatory cytokines in colostrum of nursing mothers at the extremes of reproductive age. PLOS ONE,15(6), e0231882. https://doi.org/10.1371/journal.pone.0231882

Fujimori, M., França, E. L., Fiorin, V., Morais, T. C., Honorio-França, A. C., \& de Abreu, L. C. (2015). Changes in the biochemical and immunological components of serum and colostrum of overweight and obese mothers. BMC Pregnancy and Childbirth, 15(1). https://doi.org/10.1186/s12884-015-0574-4

Freire, K., et al (2009). Fatores associados ao uso de álcool e cigarro na gestação. Revista Brasileira de Ginecologia e Obstetrícia, 31(7), 335-341. https://doi.org/10.1590/S0100-72032009000700003

Gelaleti, R., Damasceno, D., Lima, P. H., Salvadori, D. M., Calderon, I. de M., Peraçoli, J., \& Rudge, M. V. (2015). Oxidative DNA damage in diabetic and mild gestational hyperglycemic pregnant women. Diabetology \& Metabolic Syndrome,7(1), 1. https://doi.org/10.1186/1758-5996-7-1

Granot, E., Ishay-Gigi, K., Malaach, L., \& Flidel-Rimon, O. (2015). Is there a difference in breast milk fatty acid composition of mothers of preterm and term infants? The Journal of Maternal-Fetal \& Neonatal Medicine, 29(5), 832-835. https://doi.org/10.3109/14767058.2015.1020785

Gregory, K., Jackson, S., Korst, L., \& Fridman, M. (2011). Cesarean versus Vaginal Delivery: Whose Risks? Whose Benefits? American Journal of Perinatology, 29(01), 07-18. https://doi.org/10.1055/s-0031-1285829

Jaldin, M. da G. M., Pinheiro, F. S., Santos, A. M. dos, Muniz, N. C., \& Brito, L. M. O. (2011). Crescimento do perímetro cefálico nos primeiros seis meses em crianças em aleitamento materno exclusivo. Revista Paulista de Pediatria, 29(4), 509-514. https://doi.org/10.1590/s0103-05822011000400007

Kamel, R. M. (2010). The onset of human parturition. Archives of Gynecology and Obstetrics, 281(6), 975-982. https://doi.org/10.1007/s00404-010-1365-9

Khodayar-Pardo, P., Mira-Pascual, L., Collado, M. C., \& Martínez-Costa, C. (2014). Impact of lactation stage, gestational age and mode of delivery on breast milk microbiota. Journal of Perinatology, 34(8), 599-605. https://doi.org/10.1038/jp.2014.47

Knupp, V. M. de A. O., Melo, E. C. P., \& Oliveira, R. B. de. (2008). Distribuição do parto vaginal e da cesaria na no município do Rio de Janeiro no período de 2001 a 2004. Escola Anna Nery, 12(1), 39-44. https://doi.org/10.1590/s1414-81452008000100006

Kovács, A., Funke, S., Marosvolgyi, T., Burus, I., \& Decsi, T. (2005). Fatty Acids in Early Human Milk after Preterm and Full-Term Delivery.Journal of Pediatric Gastroenterology and Nutrition, 41(4), 454-459. https://doi.org/10.1097/01.mpg.0000176181.66390.54

Leal, C. A. M., Schetinger, M. R. C., Leal, D. B. R., Morsch, V. M., da Silva, A. S., Rezer, J. F. P., de Bairros, A. V., \& Jaques, J. A. dos S. (2011). Oxidative stress and antioxidant defenses in pregnant women. Redox Report, 16(6), 230-236. https://doi.org/10.1179/1351000211y.0000000013

Leguizamon Junior, T., Steffani, J. A., \& Bonamigo, E. L. (2013). Escolha da via de parto: expectativa de gestantes e obstetras. Revista Bioética, 21(3), 509517. https://doi.org/10.1590/s1983-80422013000300015

Liu, S., Liston, R. M., Joseph, K. S., Heaman, M., Sauve, R., \& Kramer, M. S. (2007). Maternal mortality and severe morbidity associated with low-risk planned cesarean delivery versus planned vaginal delivery at term. Canadian Medical Association Journal, 176(4), 455-460. https://doi.org/10.1503/cmaj.060870 
Lubetzky, R., Sever, O., Mimouni, F. B., \& Mandel, D. (2015). Human Milk Macronutrients Content: Effect of Advanced Maternal Age. Breastfeeding Medicine, 10(9), 433-436. https://doi.org/10.1089/bfm.2015.0072

Lucas, A., Gibbs, J. A., Lyster, R. L., \& Baum, J. D. (1978). Creamatocrit: simple clinical technique for estimating fat concentration and energy value of human milk. BMJ, 1(6119), 1018-1020. https://doi.org/10.1136/bmj.1.6119.1018

Mandarino, N. R., Chein, M. B. da C., Monteiro Júnior, F. das C., Brito, L. M. O., Lamy, Z. C., Nina, V. J. da S., Mochel, E. G., \& Figueiredo Neto, J. A. de. (2009). Aspectos relacionados à escolha do tipo de parto: um estudo comparativo entre uma maternidade pública e outra privada, em São Luís, Maranhão, Brasil. Cadernos de Saúde Pública, 25(7), 1587-1596. https://doi.org/10.1590/s0102-311x2009000700017

Moraes, L. L. de, Campos, L. D. A., Ferrari, D. V. de J., Saeki, E. K., Polettini, J., \& Morceli, G. (2019). Impacto da idade materna na acidez do colostro de nutrizes em maternidade do interior do Estado de São Paulo. Journal of Human Growth and Development, 29(2), 153-160. https://doi.org/10.7322/jhgd.v29.9414

Morceli, G., França, E., Magalhães, V., Damasceno, D., Calderon, I., \& Honorio-França, A. (2010). Diabetes induced immunological and biochemical changes in human colostrum. Acta Paediatrica,100(4), 550-556. https://doi.org/10.1111/j.1651-2227.2010.02070.x

Norman, J. E., Bollapragada, S., Yuan, M., \& Nelson, S. M. (2007). Inflammatory pathways in the mechanism of parturition. BMC Pregnancy and Childbirth, 7(S1). https://doi.org/10.1186/1471-2393-7-s1-s7

Polkowski, M., Kuehnle, E., Schippert, C., Kundu, S., Hillemanns, P., \& Staboulidou, I. (2017). Neonatal and Maternal Short-Term Outcome Parameters in Instrument-Assisted Vaginal Delivery Compared to Second Stage Cesarean Section in Labour: A Retrospective 11 -Year Analysis. Gynecologic and Obstetric Investigation, 83(1), 90-98. https://doi.org/10.1159/000458524

Reti, N. G., Lappas, M., Riley, C., Wlodek, M. E., Permezel, M., Walker, S., \& Rice, G. E. (2007). Why do membranes rupture at term? Evidence of increased cellular apoptosis in the supracervical fetal membranes. American Journal of Obstetrics and Gynecology, 196(5), 484.e1-484.e10. https://doi.org/10.1016/j.ajog.2007.01.021

Sassá, A. H., Schmidt, K. T., Rodrigues, B. C., Ichisato, S. M. T., Higarashi, I. H., \& Marcon, S. S. (2014). Bebês pré-termo: aleitamento materno e evolução ponderal. Revista Brasileira de Enfermagem, 67(4), 594-600. https://doi.org/10.1590/0034-7167.2014670415

Shynlova, O., Nedd-Roderique, T., Li, Y., Dorogin, A., \& Lye, S. J. (2012). Myometrial immune cells contribute to term parturition, preterm labour and postpartum involution in mice. Journal of Cellular and Molecular Medicine, 17(1), 90-102. https://doi.org/10.1111/j.1582-4934.2012.01650.x

Şimşek, Y., Karabiyik, P., Polat, K., Duran, Z., \& Polat, A. (2014). Mode of delivery changes oxidative and antioxidative properties of human milk: a prospective controlled clinical investigation. The Journal of Maternal-Fetal \& Neonatal Medicine,28(6), 734-738. https://doi.org/10.3109/14767058.2014.932345

Sinanoglou, V. J., Cavouras, D., Boutsikou, T., Briana, D. D., Lantzouraki, D. Z., Paliatsiou, S., Volaki, P., Bratakos, S., Malamitsi-Puchner, A., \& Zoumpoulakis, P. (2017). Factors affecting human colostrum fatty acid profile: A case study. PLOS ONE, $12(4)$, e0175817. https://doi.org/10.1371/journal.pone.0175817

Soares Pereira, A., Moreira Shitsuka, D., José Parreira, F., \& Shitsuka, R. (2018). METODOLOGIA DA PESQUISA CIENTÍFICA (1st ed., Vol. 1). Núcleo de Tecnologia Educacional da Universidade Federal de Santa Maria . https://repositorio.ufsm.br/bitstream/handle/1/15824/Lic_Computacao_MetodologiaPesquisa-Cientifica.pdf?sequence $=1$

Tonini, E., Broll, A. M., \& Corrêa, E. N. (2013). Avaliação do estado nutricional e hábito alimentar de funcionários de uma instituição de ensino superior do oeste de Santa Catarina. O Mundo Da Saúde, 37(3), 268-279. https://doi.org/10.15343/0104-7809.2013373268279

Wang, R., Wiemels, J. L., Metayer, C., Morimoto, L., Francis, S. S., Kadan-Lottick, N., DeWan, A. T., Zhang, Y., \& Ma, X. (2016). Cesarean Section and Risk of Childhood Acute Lymphoblastic Leukemia in a Population-Based, Record-Linkage Study in California. American Journal of Epidemiology, 185(2), 96-105. https://doi.org/10.1093/aje/kww153

Weidle, W. G., Medeiros, C. R. G., Grave, M. T. Q., \& Dal Bosco, S. M. (2014). Escolha da via de parto pela mulher: autonomia ou indução? Cadernos Saúde Coletiva, 22(1), 46-53. https://doi.org/10.1590/1414-462x201400010008 